\title{
Effect of Bifidobacterium thermophilum RBL67 and fructo-oligosaccharides on the gut microbiota in Göttingen minipigs
}

\author{
Sabine A. Tanner ${ }^{1}$, Christophe Lacroix ${ }^{1, *}$, Christophe Del'Homme ${ }^{2}$, Christoph Jans ${ }^{1}$, \\ Annina Zihler Berner ${ }^{1}$, Annick Bernalier-Donadille ${ }^{2}$ and Christophe Chassard ${ }^{1}$ \\ ${ }^{1}$ Laboratory of Food Biotechnology, Institute of Food, Nutrition and Health, ETH Zurich, Schmelzbergstrasse 7 , 8092 Zurich, \\ Switzerland \\ ${ }^{2}$ INRA, UR454 Microbiology Unit, Clermont-Ferrand Research Centre, 63122 Saint Genès-Champanelle, France \\ (Submitted 6 February 2015 - Final revision received 4 May 2015 - Accepted 20 May 2015 - First published online 21 July 2015)
}

Modulating the gut microbiota via dietary interventions is a common strategy to enhance the natural defence mechanisms of the host. Several in vitro studies have highlighted the probiotic potential of Bifidobacterium thermophilum RBL67 (RBL67) selected for its anti-Salmonella effects. The present study aimed to investigate the impact of RBL67 alone and combined with fructooligosaccharides (FOS) on the gut microbiota of Göttingen minipigs. Minipigs were fed a basal diet supplemented with $8 \mathrm{~g} / \mathrm{d}$ probiotic powder $\left(1 \times 10^{9} \mathrm{CFU} / \mathrm{g}\right.$ in skim milk matrix) (probiotic diet (PRO)), $8 \mathrm{~g} / \mathrm{d}$ probiotic powder plus $8 \mathrm{~g} / \mathrm{d}$ FOS (synbiotic diet (SYN)) or $8 \mathrm{~g} / \mathrm{d}$ skim milk powder (control), following a cross-sectional study design. Faecal and caecal microbiota compositions were analysed with pyrosequencing of 16S rRNA genes and quantitative PCR. Metabolic activity in the caecum and colon was measured by HPLC. 16S rRNA gene amplicon sequencing revealed that minipig faeces show close similarity to pig microbiota. During the treatments and at the time of killing of animals, RBL67 was consistently detected in faeces, caecum and colon at numbers of $10^{5}-10^{6} 16 \mathrm{~S}$ rRNA copies/g content after feeding PRO and SYN diets. At the time of killing of animals, significantly higher Bifidobacterium numbers in the caecum and colon of SYN-fed minipigs were measured compared with PRO. Our data indicate that the Göttingen minipig may be a suitable model for gut microbiota research in pigs. Data from this first in vivo study of RBL67 colonisation suggest that the combination with FOS may represent a valuable symbiotic strategy to increase probiotic bacteria levels and survival in gastrointestinal tracts for feed and food applications.

Key words: Probiotics: Synbiotics: Gut microbiota modulation: In vivo studies: Animal models

The gut microbiota provides a beneficial barrier function for the host, especially against common enteropathogens such as Salmonella or Escherichia coli that are encountered in pig farms. This barrier effect is possibly enhanced by probiotic bacteria that contribute to gut microbiota modulation. Specific characteristics attributed to probiotics include competition for nutrients and adhesion sites, stimulation of SCFA, production of antimicrobial substances and modulation of the immune response ${ }^{(1,2)}$. Probiotic traits are strain-specific, and must therefore be proven for any given strain. With safe history of use, strains of the genera Bifidobacterium and Lactobacillus are widely represented among probiotics $^{(2)}$. Bifidobacterium thermophilum strains have been primarily isolated from the bovine rumen and animal faeces, mainly calf and pig $^{(3,4)}$, and have also been found in the faeces of babies ${ }^{(5,6)}$

The probiotic potential of the human faecal isolate B. thermophilum RBL67 (RBL67) was investigated in several in vitro studies. Strain RBL67 produces a bacteriocin-like substance ${ }^{(5,7)}$, adheres to human intestinal cell lines ${ }^{(8)}$ and exhibits protective effects on epithelial integrity in the presence of a competing human microbiota ${ }^{(9)}$. Furthermore, RBL67 showed adaptive and competitive traits in complex human intestinal ecosystems, and it led to an inhibition of Salmonella in an in vitro continuous fermentation model of the child proximal colon ${ }^{(10)}$. RBL67 has important technological features for application as a probiotic, such as being moderately oxygen-tolerant, growing at a low $\mathrm{pH}$ of 4 and up to high temperatures of $47^{\circ} \mathrm{C}$ by reaching high cell densities ${ }^{(11)}$. Recently, the genome of RBL67 was sequenced, providing information on the genetic background of the strain $^{(12)}$. Probiotic effects can be potentiated by the use of prebiotics, a concept known as synbiotics ${ }^{(13)}$. Prebiotics are non-digestible food ingredients that largely escape digestion in the upper gastrointestinal tract that are readily fermentable in the colon and can stimulate the growth of beneficial bacteria, mainly lactobacilli and bifidobacteria, to confer a health benefit to the host ${ }^{(14)}$. Fructans such as fructo-oligosaccharides (FOS) and inulin

Abbreviations: FOS, fructo-oligosaccharides; PRO, probiotic diet; qPCR, quantitative PCR; SYN, synbiotic diet.

* Corresponding author: C. Lacroix, email christophe.lacroix@hest.ethz.ch 
are among the most widely studied and applied prebiotics ${ }^{(15)}$. Inulin was shown to specifically enhance RBL67 growth in an in vitro continuous fermentation model of the child proximal colon $^{(16)}$. FOS increased molar butyrate proportions in the caecum and proximal colon of piglets, although not affecting microbial populations $^{(17)}$. A recent study showed a synergistic effect of RBL67 and FOS for reducing Salmonella colonisation in the porcine PolyFerms in vitro model while increasing the molar butyrate ratio $^{(18,19)}$

Pigs are a close model of the human gastrointestinal tract $^{(20,21)}$ and are often affected by Salmonella infections ${ }^{(22)}$. Therefore, the anti-Salmonella effect of RBL67 is of particular interest for a targeted probiotic approach to reduce Salmonella in pigs. The Göttingen minipig holds promise as novel model for investigating the effect of nutritional compounds for food and feed applications. It has important advantages compared with pigs, including high control of breeding and origin, and is small in size, allowing easier handling and consequently reducing labour and costs ${ }^{(23,24)}$. The Göttingen minipig is already widely accepted as a non-rodent toxicology model, and extensive research of the comparative biology of minipigs to pigs and humans has been undertaken ${ }^{(25)}$. Nonetheless, information about the distal part of the intestine and gut microbiota composition of minipigs is very limited.

In this study, we aimed to study in detail the Göttingen minipig gut microbiota and test the in vivo impact of RBL67 alone or combination with FOS on the microbiota composition and metabolic activities.

\section{Methods}

\section{Ethical statement}

The minipig study was conducted at the INRA facilities of Clermont-Ferrand-Theix, France. All the experimental protocols were carried out according to the European directives on the protection of animals used for scientific purposes (2010/63/EU), and the laboratory procedures have been approved by the local ethics committee CEMEAA 02.

\section{Preparation of probiotic powder}

For production of the probiotic powder, two parallel fermentations with RBL67 (our culture collection) were carried out. Bioreactors (Multifors; INFORS HT) were filled with 1 litre sterile MRS broth (online Supplementary Table S1) supplemented with $0.05 \%$ L-cysteine hydrochloride (VWR International AG) (MRS-C). RBL67 was inoculated at $2 \%(\mathrm{v} / \mathrm{v})$ after being propagated twice overnight in the same medium. Batch fermentations were performed at $37^{\circ} \mathrm{C}$ with constant flushing of the headspace with $\mathrm{CO}_{2}$ to ensure anaerobic conditions. The $\mathrm{pH}$ was maintained at 6.0 by adding $5 \mathrm{M} \mathrm{NH}_{4} \mathrm{OH}$ with constant stirring at $150 \mathrm{rpm}$. Fermentations were stopped after $14 \mathrm{~h}$, and culture broths from the two fermentations were combined. Cells were collected by centrifugation (7000 g, 10 min, room temperature) and washed in PBS, supplemented with $0.05 \%$ L-cysteine hydrochloride. After another centrifugation step, cells were resuspended in $20 \%(\mathrm{w} / \mathrm{v})$ reconstituted skim milk (RSM; Lonza Ltd) for $1 \mathrm{~h}$ at $4^{\circ} \mathrm{C}$ under constant stirring. Cells in RSM were stored at $-80^{\circ} \mathrm{C}$ for $2 \mathrm{~d}$ before freeze-drying (Christ Alpha 1-4 LD plus; Martin Christ Gefriertrocknungsanlagen $\mathrm{GmbH})$. The dried biomass was subsequently milled to a particle size of approximately $600 \mu \mathrm{m}$ and blended with skim milk powder to yield an average cell count in the final probiotic powder of $\log 9 \cdot 36$ (SD 0.07) CFU/g. Aliquots of $8 \mathrm{~g}$, corresponding to $2 \%$ probiotic powder supply in the daily minipig diet, were prepared under vacuum and stored at $4^{\circ} \mathrm{C}$, protected from light, until usage.

\section{Animals and procedures}

The present study included eight female Göttingen minipigs (20-21 months old, average body weight $28 \mathrm{~kg}$ ), randomly allocated into two treatment groups (A and B) in a crosssectional study design (Fig. 1). All animals were housed in separate units $(1 \times 1.50 \mathrm{~m})$ in a ventilated and temperaturecontrolled room $\left(22-23^{\circ} \mathrm{C}\right)$. Animals received $400 \mathrm{~g} / \mathrm{d}$ of basal diet $(18 \%$ protein $(\mathrm{N} \times 6 \cdot 25), 2 \%$ fat, $5 \%$ cellulose and $6 \%$ ash (Porcyprima, Sanders Nutrition Animale)) before the start of the study and during the wash-out periods. During treatment periods, the basal diet was supplemented with $8 \mathrm{~g} / \mathrm{d}$ skim milk powder (control (CON)), $8 \mathrm{~g} / \mathrm{d}$ probiotic powder (probiotic diet (PRO)) or $8 \mathrm{~g} / \mathrm{d}$ probiotic powder plus $8 \mathrm{~g} / \mathrm{d}$ FOS (Fibrulose F97; Cosucra Groupe) (synbiotic diet (SYN)), according to the experimental design of the feeding trial (Fig. 1). Animals had access to water ad libitum throughout the study period. Faecal samples were collected daily and immediately stored at $-80^{\circ} \mathrm{C}$ for subsequent DNA extraction and metabolite analyses ${ }^{(26)}$.

At the end of the trial, pigs were euthanised with an injection of pentobarbital (Doléthal, $125 \mathrm{mg} / \mathrm{kg}$ body weight; Vétoquinol).

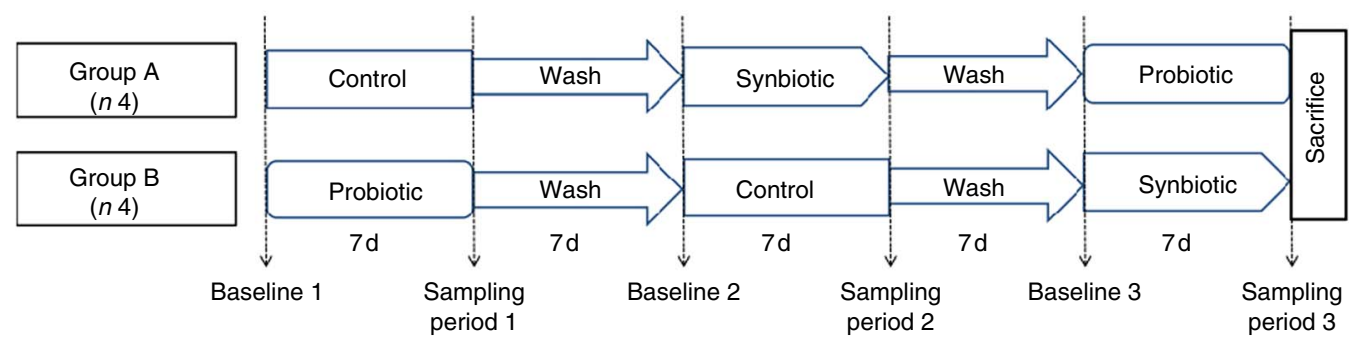

Fig. 1. Experimental set up of the feeding trial using two groups of four Göttingen minipigs. The diets included were as follows: $400 \mathrm{~g}$ of basal diet supplemented with $8 \mathrm{~g} / \mathrm{d}$ milk powder (control), $8 \mathrm{~g} / \mathrm{d}$ probiotic powder with average Bifidobacterium thermophilum RBL67 cell count of log 9.36 (sD 0.07) colony-forming units/g (probiotic) and $8 \mathrm{~g} / \mathrm{d}$ probiotic powder $+8 \mathrm{~g} / \mathrm{d}$ Fibrulose F97 (synbiotic). During wash periods, minipigs received $400 \mathrm{~g} / \mathrm{d}$ basal diet without supplement. 
Four minipigs (two minipigs of each group) were euthanised on day 36, whereas the remaining four minipigs were euthanised on day 37. After euthanisation, caecum and colon (proximal, middle and distal) contents were collected and immediately stored at $80^{\circ} \mathrm{C}$ until further analysis.

Faecal samples as well as caecum and colonic (proximal, middle and distal) contents were analysed for bacterial populations by quantitative PCR (qPCR) and for metabolite concentrations by HPLC analysis. The microbial profiles of faecal samples before the start of the study (day 1) as well as caecal content samples were also analysed for their microbial profiles with 16S rRNA gene-based amplicon sequencing.

\section{Microbial composition by quantitative polymerase chain reaction}

Genomic DNA for qPCR was extracted using the FastDNA SPIN Kit for soil (MP Biomedicals) according to the manufacturer's instructions. qPCR was performed using an ABI PRISM 7500PCR sequence detection system (Applied Biosystems) for the enumeration of predominant bacterial groups of the swine gut microbiota $^{(27)}$, including Bifidobacterium spp., Lactobacillus/ Pediococcus/Leuconostoc spp., Bacteroides-Prevotella, Enterobacteriaceae, Roseburia spp./Eubacterium rectale, Faecalibacterium prausnitzii, Streptococcus spp. and Eubacterium hallii, using specific primers (online Supplementary Table S2). Standard curves for each target group were prepared as described previously ${ }^{(28)}$, and assays were carried out using the KAPA SYBR ${ }^{\circledR}$ FAST qPCR Kit (Kapa Biosystems, Inc.) in a reaction volume of $25 \mu \mathrm{l}$. A TaqMan assay was carried out for the enumeration of $B$. thermophilum with specific primers (online Supplementary Table S2) using the RT-QP2X-03WOULR Mastermix (Eurogentec S.A.) and reaction conditions set by Mathys et al. ${ }^{(6)}$.

\section{Microbial composition by 165 rRNA gene-based amplicon sequencing}

For 16S rRNA gene-based amplicon sequencing analysis, DNA from individual faecal samples $(n 8)$ before the start of the study (day 1) and caecal samples ( $n$ ) at the end of the study was extracted using the FastDNA SPIN Kit for soil. Extracted DNA was sent to DNAVision for high-throughput sequencing of the hypervariable V5-V6 region of the entire 16S rRNA gene pool using a 454 Life Sciences Genome Sequencer FLX instrument (Roche AG), according to procedures described previously ${ }^{(29)}$. Resulting sequencing reads were quality-filtered according to the following three criteria: maximum of one mismatch in barcode and primers, at least 240 nucleotides length and a maximum of two undetermined bases per sequence. Sequencing reads passing the quality check were assigned at the phylum, family and genus level using the Ribosomal Database Project (RDP) Bayesian classifier (v2.1) ${ }^{(30)}$ with a confidence threshold of $80 \%$. Sequences were further assigned into operational taxonomic units (OTU) based on nearest neighbour clustering using mothur software package ${ }^{(31)}$. Chao1 richness and Shannon diversity were calculated based on the numbers of OTU. The complete 16S rRNA gene-based amplicon sequencing data set has been deposited to the National Center for Biotechnology Information Sequence Read Archive under accession number SRP044704.

\section{Metabolite analysis}

Samples for HPLC analyses were prepared according to the protocol described by Dostal et al. ${ }^{(32)}$. In brief, caecum and colonic content (proximal, middle and distal) samples (100$200 \mathrm{mg}$ ) were homogenised with $1 \mathrm{ml}$ of $0 \cdot 15 \mathrm{M} \mathrm{H}_{2} \mathrm{SO}_{4}$, centrifuged $\left(4^{\circ} \mathrm{C}, 9000 \mathbf{g}, 20 \mathrm{~min}\right)$ and the resulting supernatants were filtered through a $0.45 \mu \mathrm{m}$ nylon filter (Infochroma AG) before injection. Total metabolites (acetate, propionate, butyrate, valerate, isovalerate, isobutyrate, formate and lactate) as well as glucose and lactose were quantified in duplicate by HPLC (LaChrome; Hitachi High-Tech) using an Aminex HPX-87H column (Bio-Rad Laboratories AG) at a flow rate of $0.4 \mathrm{ml} / \mathrm{min}$ and with $10 \mathrm{~mm}_{2} \mathrm{SO}_{4}$ as the eluent. Data are expressed as means from duplicate analyses in mm/g caecal and colonic contents.

\section{Statistical analysis}

All the statistical analyses were performed using PASW Statistics for Windows (v.18.0; SPSS, Inc.). qPCR data were $\log _{10}$ transformed before analysis. To test the effects of experimental diets in faecal samples, mean qPCR data of group A $(n 4)$ and $\mathrm{B}(n 4)$ at the end of each treatment period (Fig. 1) were compared using ANCOVA. The bacterial target group was treated as a dependent factor, the treatment group as an independent factor and the baseline of the corresponding treatment period (Fig. 1) was taken as the covariate. QPCR data from faecal samples are expressed as mean values with their standard errors from four minipigs of group A $(n$ 4) and $\mathrm{B}(n$ 4).

A factorial repeated measures ANOVA was performed to test the effects of experimental diets (between-subjects factor; PRO and SYN), intestinal segment (within-subject factor: caecum, proximal, middle and distal colon) and their interactions on bacterial groups targeted by qPCR and metabolite concentrations in the caecum and colon. If assumption of sphericity was not met, the Greenhouse-Geisser correction was applied and significant effects of intestinal segments were further tested using pairwise comparison, with post hoc Bonferroni's correction for multiple comparisons.

In order to compare differences in microbial composition and diversity using $16 \mathrm{~S}$ rRNA gene-based amplicon sequencing, the relative abundance data at genus and family levels from PRO and SYN in the caecum at the time of killing of animals were compared using the non-parametric Mann-Whitney $U$ test with exact significance.

qPCR, HPLC and 16S rRNA gene-based amplicon sequencing data from caecum and colon are expressed as mean with standard deviation for PRO $(n$ 4) and SYN $(n 4)$.

For all statistical tests, results were considered significant at $P \leq 0 \cdot 05$. 


\section{Results}

Faecal microbiota composition and diversity of Göttingen minipigs

Faecal microbiota composition and diversity were assessed before the start of the study (baseline 1) with qPCR targeting predominant bacterial groups of the swine gut microbiota and 16S rRNA gene-based amplicon sequencing. At baseline 1, the mean $(n 8)$ total $16 \mathrm{~S}$ rRNA gene copy number by qPCR was log 11.8 (sD 0.2) copies/g faeces (Table 1). The most abundant bacterial groups targeted were Bacteroides-Prevotella $(\log 10 \cdot 9$ (sD 0.2) copies/g) and Enterobacteriaceae (log 10.9 (SD 0.7) copies/g), followed by Lactobacillus/Leuconostoc/Pediococcus $(\log 10.3$ (sD 0.4) copies/g), Streptococcus (log 9.6 (sD 0.6) copies/g), F. prausnitzii (log 9.3 (sD 0.2) copies/g), E. hallii (log $9 \cdot 3$ (sD 0.2 ) copies/g) and Roseburia/E. rectale (log 8.5 (sD $0 \cdot 3$ ) copies/g). Bifidobacterium was least abundant with $\log 7 \cdot 4$ (SD 0.2) copies/g, whereas B. thermophilum was not detected in both groups at baseline.

Microbiome analysis on the V5-V6 region of the entire 16S rRNA gene pool from faecal samples of minipigs ( $n$ 8) before the start of the study generated a total of 72048 quality-filtered reads with a mean of 9991 (sD 3864) reads/sample and a mean read length of 258 (sD 1) bp. Richness (Chao1 index: 2369 (SD 769)) and diversity (non-parametric Shannon index: 4.50 (SD $0 \cdot 88)$ ) were estimated from the number of OTU with a similar cut-off of $0.03 \%$ (1068 (SD 329)).

At the phylum level, mean relative abundance data revealed the predominance of four major phyla (Fig. 2). The phylum Firmicutes was the most abundant (approximately 75\%) followed by Bacteroidetes (approximately 14\%), Proteobacteria (approximately $4 \%$ ) and Spirochaetes (approximately 3\%), whereas $4 \%$ of the reads could not be assigned to any phylum. Inter-individual variation was observed in all four phyla, mostly in Bacteroidetes and Proteobacteria, with relative abundances ranging from 4 to $22 \%$ and from $0 \cdot 16$ to $17 \%$, respectively. At the family level, the most abundant phylum, Firmicutes, encompassed the families Streptococcaceae (25\%), Ruminococcaceae (11\%), Lactobacillaceae (8\%), Clostridiaceae (5\%), Lachnospiraceae (4\%) and Erysipelotrichaceae (4\%). The phylum Bacteroidetes was almost exclusively represented by the family Prevotellaceae (7\%), whereas Porphyromonadaceae and Rikenellaceae, the two other families detected from this phylum, displayed low relative abundances (both at $1 \%$ ). Two other families, Enterobacteriaceae (phylum Proteobacteria) and Spirochaetaceae (phylum Spirochaetes), were detected (both approximately $3 \%$ ), and unclassified reads accounted for approximately $24 \%$ of all reads at the family level. In all, eleven predominant genera represented at least $1 \%$ relative abundance in at least one minipig, whereas unclassified reads accounted for $44 \%$ (Fig. 3). The highest relative abundance was observed for the genera Streptococcus (25\%), Lactobacillus (8\%) and Clostridium (5\%). The genus Acinetobacter was detected in only one pig (pig B7), whereas the genus Escherichia/Shigella was missing in one pig (pig A2) out of eight. The remaining nine genera were detected in all minipigs. At the genus level, inter-individual variations were observed, most prominently within the genera Streptococcus, Lactobacillus and Clostridium.

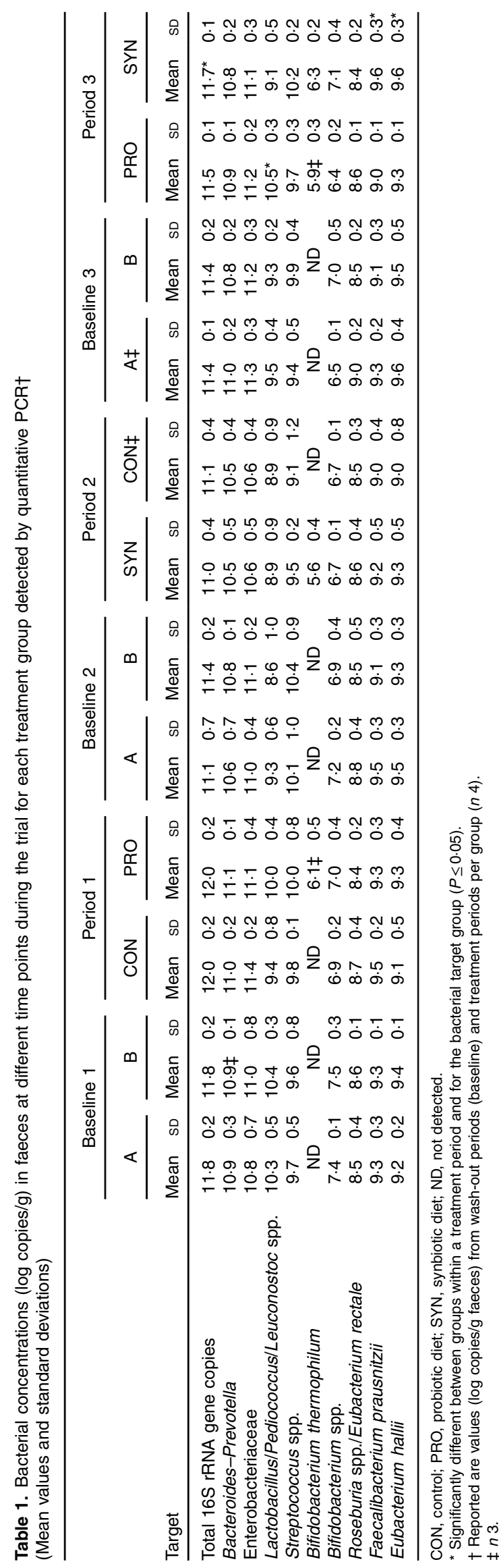



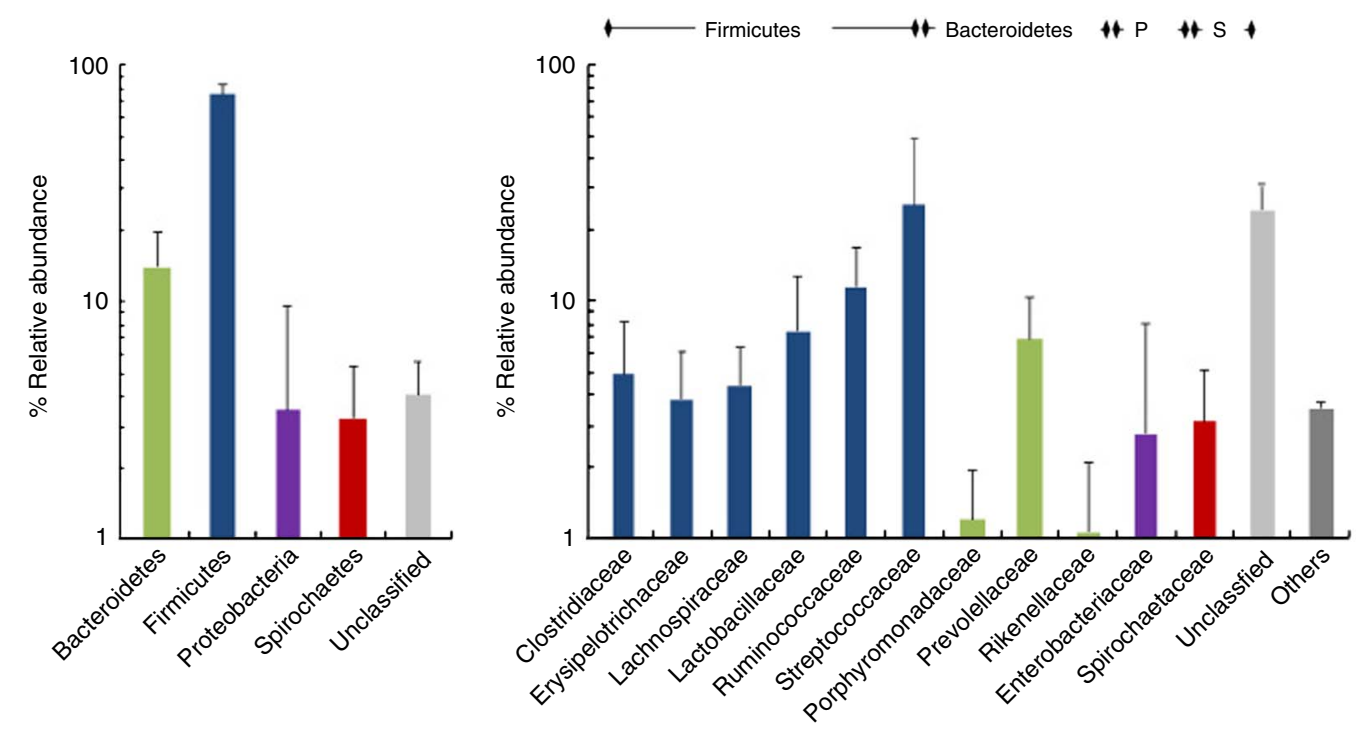

Fig. 2. Mean relative $16 \mathrm{~S}$ rRNA gene abundances detected in faeces from Göttingen minipigs at the phylum and family level using 454 pyrosequencing. Values are means with standard deviation for all minipigs $(n$ ) before the start of the study (baseline 1 ) and with a relative abundance $>1 \%$ in at least one minipig. Relative abundances $<1 \%$ are summarised in the group 'others'. P, Proteobacteria; S, Spirochaetes.

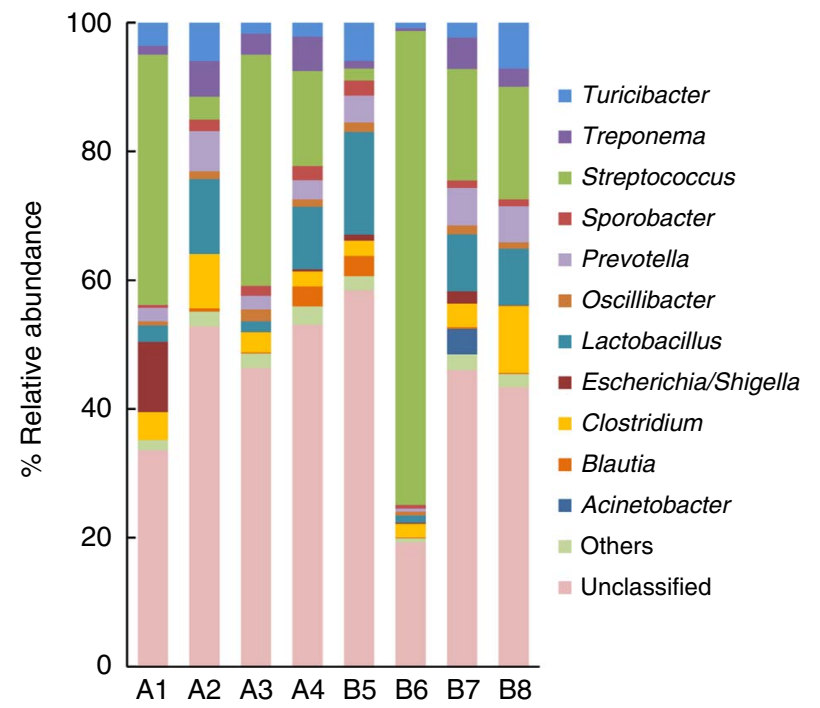

Fig. 3. Relative $16 \mathrm{~S}$ rRNA gene abundance detected in faeces from Göttingen minipigs at the genus level using 454 pyrosequencing. Values are given for each minipig individually before the start of the study (baseline 1). Relative abundances $<1 \%$ are summarised in the group 'others'.

\section{Effect of probiotic and synbiotic diets on faecal bacterial concentrations}

Differences in faecal bacterial concentrations upon feeding experimental diets PRO or SYN were assessed by qPCR targeting predominant bacterial groups of the swine microbiota. Throughout the study (during wash-out and treatment periods), high and stable concentrations of total 16S rRNA gene copies ( $\log 11.5$ (SD 0.4) copies/g faeces), Enterobacteriaceae ( $\log 11 \cdot 0$ (SD 0.3) copies/g faeces) and Bacteroides-Prevotella (log $10 \cdot 8$ (SD $0 \cdot 2$ ) copies/g faeces) were measured in both groups. Stable concentrations during wash-out and treatment periods were also observed for Streptococcus (log 9.8 (sD 0.4) copies/g faeces), Roseburia/E. rectale ( $\log 8 \cdot 6$ (sD $0 \cdot 2$ ) copies/g faeces) and Bifidobacterium (log 6.9 (SD 0.3) copies/g faeces). B. thermophilum was not detected during wash-out periods and in the CON groups, but was detected in the treatment groups that included RBL67 (PRO or SYN). Experimental diets in periods 1 and 2 did not significantly change faecal bacterial numbers of the groups targeted by qPCR compared with the CON group. In period 3, faecal Lactobacillus/Pediococcus/Leuconostoc numbers were significantly higher with PRO (log $10 \cdot 5$ (SD 0.3) copies/g) compared with SYN (log 9.1 (SD 0.5) copies/g) $(P=0.007)$. In contrast, total $16 \mathrm{~S}$ rRNA $(P=0.011), F$. prausnitzii $(P=0.001)$ and $E$. hallii $(P=0.043)$ numbers were significantly higher with SYN compared with PRO in the third treatment period.

\section{Effects of probiotic and synbiotic diets on bacterial concentration and composition in the caecum and colon}

The effect of experimental diets (PRO and SYN) on bacterial composition in the caecum and colon was assessed with qPCR and 16S rRNA gene-based amplicon sequencing (Table 2, Fig. 4). A significant effect of experimental diets in the caecum and colon was observed for Bifidobacterium $(P=0.021)$ with higher numbers in the SYN group compared with the PRO group. For all the other bacterial groups (total 16S rRNA gene copies, Bacteroides-Prevotella, Enterobacteriaceae, Streptococcus, B. thermophilum, Lactobacillus/Leuconostoc/ Pediococcus, Roseburia/E. rectale, $F$. prausnitzii and $E$. hallii), no significant effect of experimental diets (PRO and SYN) was observed for bacterial gene copy numbers in the caecum and the different colon segments.

Moreover, 16S rRNA gene-based amplicon sequencing indicated significantly $(P \leq 0.05)$ lower relative abundance of 


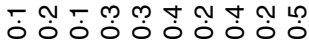
T人t.

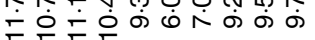

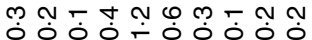
L $0-0 m a 0 m+0$ 둥ㄷㄷ응

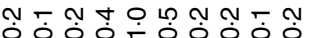

$\operatorname{dog}$

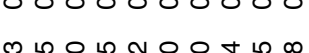

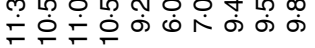

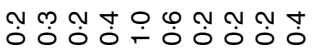

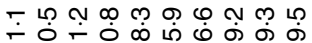

ก்َ is

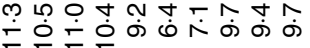

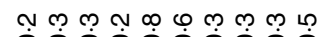
- + $-\infty \circ \infty$

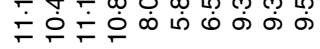

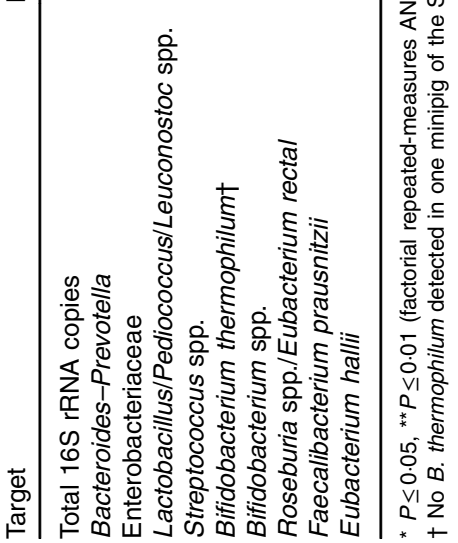

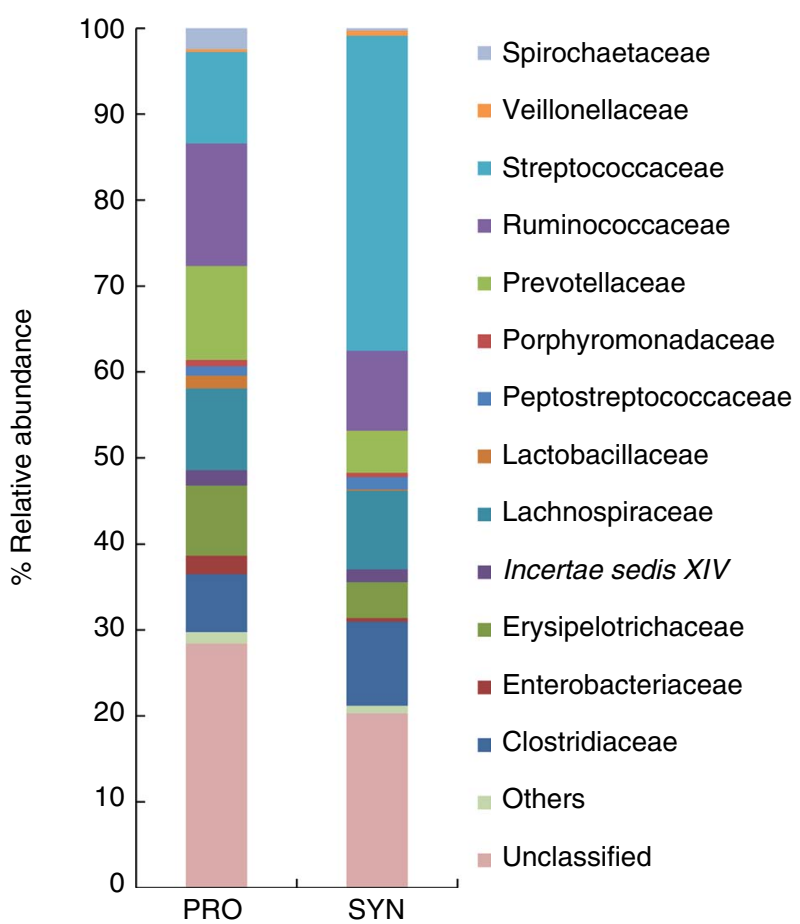

Fig. 4. Caecal microbiota composition of minipigs in the probiotic (PRO, $n 4$ ) and synbiotic (SYN, $n 4$ ) groups analysed by 454 pyrosequencing. Relative abundance is depicted at the family level. Families $<1 \%$ are summarised in the group 'others'.

Lactobacillaceae (0.15\%) and Spirochaetaceae (0.27\%) for minipigs from the SYN group compared with the PRO group (Lactobacillaceae 1.48\%; Spirochaetaceae 2.47\%) (Fig. 4).

Intestinal segments displayed a statistically significant difference in the total 16S rRNA gene copies $(P=0 \cdot 004)$, B. thermophilum $(P=0.005)$ and Roseburia/E. rectale $(P=0.028)$. Post hoc pairwise comparisons revealed lower total $16 \mathrm{~S}$ rRNA gene copies in the first two segments (caecum and proximal colon) compared with the third (middle colon), whereas $B$. thermophilum concentrations were higher in the caecum compared with the middle colon. For Roseburia/E. rectale pairwise comparisons failed to detect the location effects. For all the bacterial groups, no significant interaction between experimental diets and intestinal segments was observed.

\section{Effect of probiotic and synbiotic diets on microbial activity in the caecum and colon}

Metabolite concentrations were analysed in the caecum and colon content samples from PRO ( $n$ 4) and SYN $(n$ 4) at the time of killing of animals by HPLC. Total SCFA and measured metabolite concentrations did not significantly differ between the two experimental diets (Fig. 5, online Supplementary Table S3). A significant effect of the intestinal segment was recorded for total SCFA $(P=0.029)$, acetate $(P=0.032)$, propionate $(P=0.043)$ and butyrate $(P=0.029)$, reflecting a higher metabolic activity in the caecum (total mean metabolite 
(a)

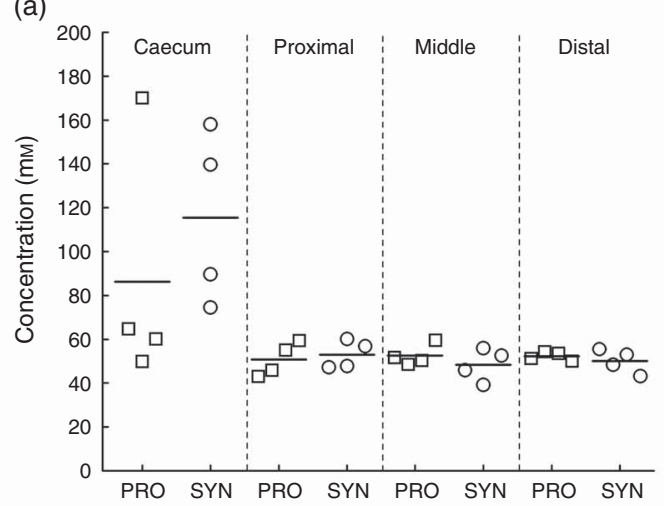

(c)

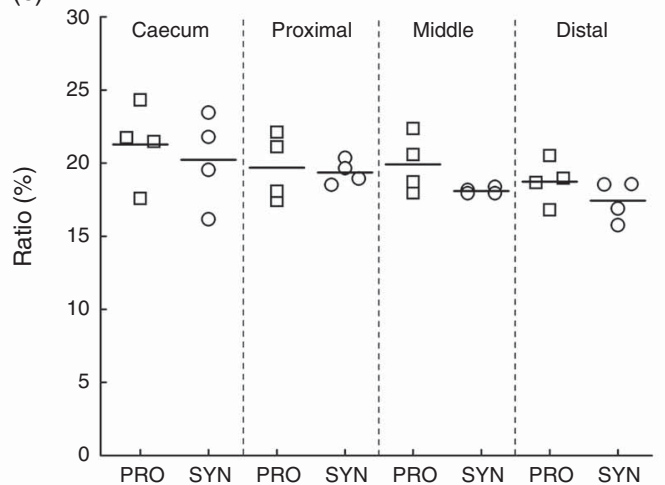

(b)

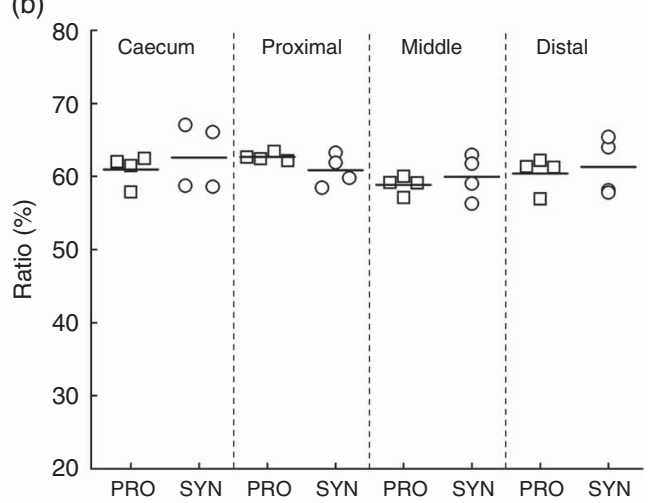

(d)

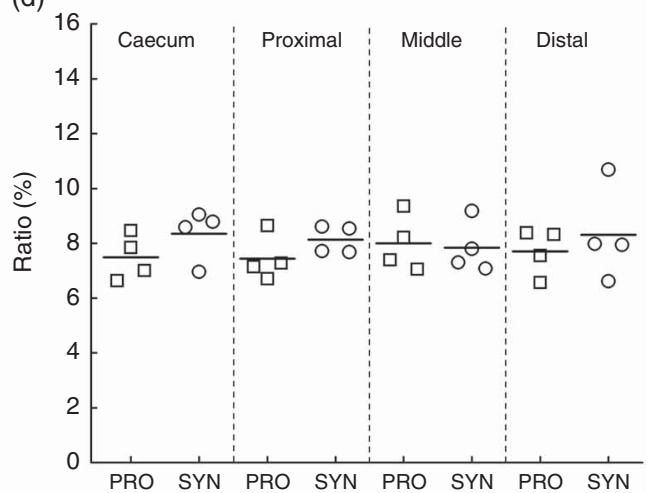

Fig. 5. Total metabolite concentrations ( $\mathrm{mm}$ ) and metabolite ratios (\% of total metabolites) detected in the caecum and subsequent colon sections (proximal, middle and distal) in probiotic (PRO) and synbiotic (SYN) groups after killing of animals using HPLC. (a) Total metabolite concentration, (b) acetate ratio, (c) propionate ratio and (d) butyrate ratio. Depicted are mean values (line) and individual values ( $\square$ PRO and O SYN).

production of 86 ( $\mathrm{SD}$ 56) $\mathrm{mm}$ for PRO and 115 (SD 40) $\mathrm{mm}$ for SYN), but also large inter-individual variations within groups. In the proximal colon, total metabolite concentrations decreased to 51 (SD 8) $\mathrm{mm}(\mathrm{PRO})$ and $53(\mathrm{SD} 6) \mathrm{mm}(\mathrm{SYN})$ and remained stable in the middle and distal colon regions. The ratios of SCFA were similar for PRO and SYN (acetate 61\%:propionate 19\%: butyrate $8 \%$ ) and remained stable along the caecum and colon sections.

Minor metabolites, isobutyrate, isovalerate and valerate also exhibited similar ratios for PRO and SYN in the caecum and colon sections (online Supplementary Table S3). Interestingly, a considerable amount of residual glucose in the caecum of one minipig from PRO (17 mm) and two from SYN (17 and $8 \mathrm{~mm}$ ) was detected, which also displayed the highest total metabolite concentration

\section{Discussion}

The present study investigated the impact of the probiotic RBL67 alone or in combination with FOS on faecal, caecal and colonic microbiota composition and activity in Göttingen minipigs, a potential new model for characterising the effect of nutritional compounds for feed and food applications on the gut microbiota and gut health.

The Göttingen minipig is particularly advantageous over conventional pig models due to its small size, facilitating handling and reducing costs ${ }^{(24)}$, and its high control in breeding and origin $^{(23)}$. However, information on the gut microbiota composition of Göttingen minipigs is scarce. Therefore, in this study, microbiota composition and activity in faeces and different colonic sections were measured using $\mathrm{qPCR}$ and next generation sequencing methods and HPLC, respectively. The results at baseline obtained by $\mathrm{qPCR}$ and $16 \mathrm{~S}$ rRNA gene-based amplicon sequencing showed that members of the Firmicutes (Streptococcus, F. prausnitzii, E. hallii, Roseburia/E. rectale, Lactobacillus/ Leuconostoc/Pediococcus), Bacteroidetes (Bacteroides-Prevotella) and Proteobacteria phylum (Enterobacteriaceae) were dominant. However, quantitative comparison between qPCR and 16S rRNA gene-based amplicon sequencing should be considered with caution due to inherent biases associated with each method. For instance, the $16 \mathrm{~S}$ rRNA gene copy number is affected by primer specificity bias in qPCR and a relatively low sensitivity is associated with 16S rRNA gene-based amplicon sequencing. As such, Bifidobacterium with the lowest abundance in qPCR likely fell below the detection limit of 16S rRNA gene-based amplicon sequencing. Thus, qPCR targeting specific populations and $16 \mathrm{~S}$ rRNA gene-based amplicon sequencing should be used in a complementary approach to accurately analyse microbiota composition. In general, the faecal microbiota composition in Göttingen minipigs revealed by $16 \mathrm{~S}$ rRNA gene-based amplicon sequencing was in accordance with recent data of the same minipig model ${ }^{(33)}$. Minor differences between the studies can be explained by the 
site-specificity of the samples (ileum, caecum, colon $v$. faeces), variations in diet and environment and methodological differences of the two studies (different variable 16S rRNA region targeted, different pipeline for reads assignment) ${ }^{(34)}$.

Our samples displayed higher relative abundance of $75 \%$ Firmicutes compared with $49 \%$ revealed by Pedersen et al. ${ }^{(33)}$. Large variations in relative abundance of Firmicutes have also been reported in studies characterising the caecal microbiota of weanling pigs ranging from approximately $37 \%{ }^{(35)}$ to up to approximately $75 \%{ }^{(36)}$. Further, in our study, lower relative abundances of Spirochaetes $(3.2 v .6 \%)$ and Tenericutes $(<1 v$. $3.8 \%$ ) but higher relative abundance of Proteobacteria (3.5 $v$. $<1 \%)$ were observed compared with the study by Pedersen et $a l^{(33)}$. At the family and genus level, our study revealed a similar microbiota composition in minipigs ${ }^{(33)}$ and pigs $^{(35-39)}$. A remarkable high relative abundance of the genus Streptococcus was recorded in three out of eight faecal samples, which is in accordance with several other pig studies ${ }^{(37-39)}$. In contrast, Pedersen et $a l^{(33)}$ and other studies on pig caecal microbiota $^{(35-39)}$ did not find a predominance of the genus Streptococcus in the caecum, suggesting a dominance of streptococci in faecal material but not in the proximal colon. The high fraction of unclassified reads (up to $58 \%$ of total) presumes that a large fraction of bacteria of the faecal microbiota in minipigs is not yet deciphered, which is in contrast with the well-studied pig microbiota. Therefore, to support the use of the minipigs as model for gut microbiota research, the microbiota composition of minipigs should be further deeply studied using culturedependent approaches for isolation of potential new microbes and culture-independent approaches using long-length amplicons. In our study, B. thermophilum was not detected in minipig faeces in the CON groups and during wash-out periods. Consequently, we assume that the general B. thermophilum population is below detection limit or absent, and data of the qPCR test used to detect $B$. thermophilum enumerated the supplemented strain RBL67. After feeding experimental diets (PRO and SYN), RBL67 was consistently detected in faecal, caecal and colonic content. Enterococcus faecium NCIMB 10415, an authorised probiotic strain for use in pig feed in the European Union ${ }^{(40)}$, was recovered at $1.8 \times 10^{5} \mathrm{CFU} / \mathrm{g}$ wet weight faeces in pregnant sows fed the probiotic for $80 \mathrm{~d}$ at approximately $1 \times 10^{9} \mathrm{CFU} / \mathrm{kg}$ feed $^{(40,41)}$, which is consistent with our data on $B$. thermophilum, despite the largely different physiological features of these two strains.

The SYN group displayed significantly higher total Bifidobacterium numbers in the caecum and colon compared with the PRO group. In addition, the analysis of $B$. thermophilum after killing of animals revealed highest numbers of $B$. thermophilum in the caecum of the SYN group. These data suggest that RBL67 is viable and growth in the caecum is stimulated by the concomitant administration of FOS, implying the synbiotic concept. The simultaneous stimulation of Bifidobacterium and RBL67 by SYN explains why the relative percentage of $B$. thermophilum in the caecum as a proportion of the total Bifidobacterium population is higher in the PRO group (36\%) compared with the SYN group (22\%). FOS has previously been reported to selectively stimulate the growth of bifidobacteria $^{(42)}$, although it has been established nowadays that other bacteria (e.g. members of the genera Roseburia, Bacteroides, Salmonella) are able to utilise prebiotics as well $^{(15,43,44)}$.

No differences between PRO and SYN were observed in metabolite concentrations measured by HPLC of the caecum and colonic samples. This is in agreement with a previous study where piglets had ad libitum access to experimental diets including $10 \mathrm{~g} / \mathrm{kg}$ FOS or transgalactooligosaccharide ${ }^{(45)}$. However, other studies have reported a significant increase in butyrate and decrease in acetate concentrations when including $4 \% \mathrm{FOS}^{(17)}$ or $3 \%$ inulin to piglet diets ${ }^{(46)}$. Different factors including prebiotic substrate (degree of polymerisation, inclusion rate), feeding frequency (ad libitum, restricted), pig breed and basal diet ${ }^{(42)}$ may contribute to the variable outcome of the different studies and complicate direct comparisons between studies. SCFA concentrations do not provide a direct marker of metabolic activity due to their rapid absorption by the host or utilisation by other members of the gut microbiota ${ }^{(46,47)}$. The rapid absorption of SCFA is demonstrated by a decrease of SCFA concentrations from the caecum to the proximal colon, as reported previously ${ }^{(48)}$ and also shown in our study. It is possible that differences in absorption and high inter-individual variations of SCFA profiles have masked the effect of FOS on metabolite concentrations, leading to non-significant differences between treatment groups. Another hypothesis may be that FOS is partly digested in the upper gastrointestinal tract, as previously shown by Loh et ${ }^{(46)}{ }^{(46)}$, where $20-50 \%$ of the supplemented inulin was digested in the jejunum of pigs.

In conclusion, Göttingen minipigs may be a suitable model for pig microbiota study, although the high fraction of assigned reads at the family and genus levels emphasise the need for additional characterisation of gut microbe diversity. We demonstrated for the first time the in vivo recovery of the probiotic candidate RBL67 by qPCR upon feeding minipigs, with a stimulation effect of FOS on the bifidobacteria population and specifically RBL67 in the caecum. Our data suggest that Göttingen minipigs can be a suitable model for investigating the impact of feed and food additives for gut health. Additional in vivo investigations using Salmonella-infected pigs must be carried out to confirm the antimicrobial efficacy of the symbiotic formulation of RBL67 and FOS against enteropathogens.

\section{Acknowledgements}

The authors thank Dr Franck Grattepanche and Friederike Plata Gröber from ETH Zurich for probiotic powder optimisation and production. They also thank Eve Delmas and Benoit Cohade from INRA in Clermont-Ferrand for assistance in sampling and animal care throughout the study.

This work was supported by the Commission for Technology and Innovation (project number 11962.1).

The authors' contributions are as follows: S. A. T., C. C., A. B.-D., C. D. and C. L. designed the study; S. A. T., C. C., C. J. and C. D. carried out the study; S. A. T. and C. C. analysed the data; S. A. T. wrote the manuscript; C. C., A. B.-D., A. Z. B., C. J., C. D. and C. L. edited and approved the manuscript.

None of the authors have any conflicts of interest to declare. 


\section{Supplementary Material}

For supplementary material/s referred to in this article ${ }^{(49-53)}$, please visit http://dx.doi.org/10.1017/S0007114515002263

\section{References}

1. Ohashi Y \& Ushida K (2009) Health-beneficial effects of probiotics: its mode of action. Anim Sci J 80, 361-371.

2. Gaggia F, Mattarelli P \& Biavati B (2010) Probiotics and prebiotics in animal feeding for safe food production. Int $J$ Food Microbiol 141, Suppl. 1, 15-28.

3. Biavati B \& Mattarelli P (2009) Genus I. Bifidobacterium. In Bergey's Manual of Systematic Bacteriology: The Actinobacteria, 2nd ed., vol. 5, pp. 171-206 [WB Whitman, P Kämpfer, M Goodfellow, GM Garrity and W Ludwig, editors]. New York, NY: Springer Verlag.

4. Gavini F, Pourcher AM, Neut C, et al. (1991) Phenotypic differentiation of bifidobacteria of human and animal origins. Int J Syst Bacteriol 41, 548-557.

5. Toure R, Kheadr E, Lacroix C, et al. (2003) Production of antibacterial substances by bifidobacterial isolates from infant stool active against Listeria monocytogenes. I Appl Microbiol 95, 1058-1069.

6. Mathys S, Lacroix C, Mini R, et al. (2008) PCR and real-time PCR primers developed for detection and identification of Bifidobacterium thermophilum in faeces. BMC Microbiol 8, 179 .

7. von Ah U (2006) Identification of Bifidobacterium thermophilum RBL67 isolated from baby feces and partial purification of its bacteriocin. PhD Thesis, ETH Zurich, Switzerland.

8. Moroni O, Kheadr E, Boutin Y, et al. (2006) Inactivation of adhesion and invasion of food-borne Listeria monocytogenes by bacteriocin-producing Bifidobacterium strains of human origin. Appl Environ Microbiol 72, 6894-6901.

9. Zihler A, Gagnon M, Chassard C, et al. (2011) Protective effect of probiotics on Salmonella infectivity assessed with combined in vitro gut fermentation-cellular models. BMC Microbiol 11, 264.

10. Zihler A, Le Blay G, Chassard C, et al. (2014) Bifidobacterium thermophilum RBL67 inhibits Salmonella enterica serovar Typhimurium in an in vitro intestinal fermentation model. J Food Nutr Disor, S1-003.

11. von Ah U, Mozzetti V, Lacroix C, et al. (2007) Classification of a moderately oxygen-tolerant isolate from baby faeces as Bifidobacterium thermophilum. BMC Microbiol 7, 79.

12. Jans C, Lacroix C, Follador R, et al. (2013) Complete genome sequence of the probiotic Bifidobacterium thermophilum strain RBL67. Genome Announc 1, e00191-00113.

13. Gibson GR \& Roberfroid MB (1995) Dietary modulation of the human colonic microbiota: introducing the concept of prebiotics. J Nutr 125, 1401-1412.

14. Gibson GR, Probert HM, Loo JV, et al. (2004) Dietary modulation of the human colonic microbiota: updating the concept of prebiotics. Nutr Res Rev 17, 259-275.

15. Scott KP, Martin JC, Duncan SH, et al. (2013) Prebiotic stimulation of human colonic butyrate-producing bacteria and bifidobacteria, in vitro. FEMS Microbiol Ecol 87, 30-40.

16. Zihler A, Gagnon M, Chassard C, et al. (2010) Unexpected consequences of administering bacteriocinogenic probiotic strains for Salmonella populations, revealed by an in vitro colonic model of the child gut. Microbiology 156, 3342-3353.

17. Mikkelsen LL \& Jensen BB (2004) Effect of fructooligosaccharides and transgalacto-oligosaccharides on microbial populations and microbial activity in the gastrointestinal tract of piglets post-weaning. Anim Feed Sci Technol 117, 107-119.
18. Tanner SA, Zihler Berner A, Rigozzi E, et al. (2014) In vitro continuous fermentation model (PolyFerms) of the swine proximal colon for simultaneous testing on the same gut microbiota. PLOS ONE 9, e94123.

19. Tanner SA, Chassard C, Berner AZ, et al. (2014) Synergistic effects of Bifidobacterium thermophilum RBL67 and selected prebiotics on inhibition of Salmonella colonization in the swine proximal colon PolyFerms model. Gut Pathog 6, 44.

20. Heinritz SN, Mosenthin R \& Weiss E (2013) Use of pigs as a potential model for research into dietary modulation of the human gut microbiota. Nutr Res Rev 26, 191-209.

21. Guilloteau P, Zabielski R, Hammon HM, et al. (2010) Nutritional programming of gastrointestinal tract development. Is the pig a good model for man? Nutr Res Rev 23, 4-22.

22. EFSA (2009) Analysis of the baseline survey on the prevalence of Salmonella in holdings with breeding pigs in the EU, 2008. EFSA J 7, 1377-1470.

23. Forster R, Ancian P, Fredholm M, et al. (2010) The minipig as a platform for new technologies in toxicology. J Pharmacol Toxicol Methods 62, 227-235.

24. Kohn F, Sharifi AR \& Simianer H (2007) Modeling the growth of the Goettingen minipig. J Anim Sci 85, 84-92.

25. Bode G, Clausing P, Gervais F, et al. (2010) The utility of the minipig as an animal model in regulatory toxicology. J Pharmacol Toxicol Methods 62, 196-220.

26. Dostal A, Lacroix C, Pham VT, et al. (2014) Iron supplementation promotes gut microbiota metabolic activity but not colitis markers in human gut microbiota-associated rats. $\mathrm{BrJ}$ Nutr 111, 2135-2145.

27. Leser TD, Amenuvor JZ, Jensen TK, et al. (2002) Cultureindependent analysis of gut bacteria: the pig gastrointestinal tract microbiota revisited. Appl Environ Microbiol 68, 673-690.

28. Dostal A, Fehlbaum S, Chassard C, et al. (2013) Low iron availability in continuous in vitro colonic fermentations induces strong dysbiosis of the child gut microbial consortium and a decrease in main metabolites. FEMS Microbiol Ecol 83, 161-175.

29. Jost T, Lacroix C, Braegger C, et al. (2013) Assessment of bacterial diversity in breast milk using culture-dependent and culture-independent approaches. Br J Nutr 110, 1253-1262.

30. Wang Q, Garrity GM, Tiedje JM, et al. (2007) Naive Bayesian classifier for rapid assignment of rRNA sequences into the new bacterial taxonomy. Appl Environ Microbiol 73, 5261-5267.

31. Schloss PD, Westcott SL, Ryabin T, et al. (2009) Introducing mothur: open-source, platform-independent, communitysupported software for describing and comparing microbial communities. Appl Environ Microbiol 75, 7537-7541.

32. Dostal A, Baumgartner J, Riesen N, et al. (2014) Effects of iron supplementation on dominant bacterial groups in the gut, faecal SCFA and gut inflammation: a randomised, placebocontrolled intervention trial in South African children. $\mathrm{Br} \mathrm{J}$ Nutr 112, 547-556.

33. Pedersen R, Ingerslev HC, Sturek M, et al. (2013) Characterisation of gut microbiota in Ossabaw and Gottingen minipigs as models of obesity and metabolic syndrome. PLOS ONE 8, e56612.

34. Claesson MJ \& O'Toole PW (2010) Evaluating the latest highthroughput molecular techniques for the exploration of microbial gut communities. Gut Microbes 1, 277-278.

35. Poroyko V, White JR, Wang M, et al. (2010) Gut microbial gene expression in mother-fed and formula-fed piglets. PLOS ONE 5, e12459.

36. Buzoianu SG, Walsh MC, Rea MC, et al. (2012) Highthroughput sequence-based analysis of the intestinal microbiota of weanling pigs fed genetically modified MON810 maize expressing Bacillus thuringiensis Cry1Ab (Bt maize) for 31 days. Appl Environ Microbiol 78, 4217-4224. 
37. Kim HB, Borewicz K, White BA, et al. (2011) Longitudinal investigation of the age-related bacterial diversity in the feces of commercial pigs. Vet Microbiol 153, 124-133.

38. Lamendella R, Domingo JW, Ghosh S, et al. (2011) Comparative fecal metagenomics unveils unique functional capacity of the swine gut. BMC Microbiol 11, 103.

39. Looft T, Allen HK, Cantarel BL, et al. (2014) Bacteria, phages and pigs: the effects of in-feed antibiotics on the microbiome at different gut locations. ISME J 8, 1566-1576.

40. Simon O (2005) Micro-organisms as feed additives-probiotics. Adv Pork Prod 16, 161-167.

41. Macha M, Taras D, Vahjen W, et al. (2004) Specific enumeration of the probiotic strain Enterococcus faecium NCIMB 10415 in the intestinal tract and in faeces of piglets and sows. Arch Anim Nutr 58, 443-452.

42. Flickinger EA, Van Loo J \& Fahey GC Jr (2003) Nutritional responses to the presence of inulin and oligofructose in the diets of domesticated animals: a review. Crit Rev Food Sci Nutr 43, 19-60.

43. van der Meulen R, Makras L, Verbrugghe K, et al. (2006) In vitro kinetic analysis of oligofructose consumption by Bacteroides and Bifidobacterium spp. indicates different degradation mechanisms. Appl Environ Microbiol $\mathbf{7 2}$, 1006-1012.

44. Martin-Pelaez S, Gibson GR, Martin-Orue SM, et al. (2008) In vitro fermentation of carbohydrates by porcine faecal inocula and their influence on Salmonella typhimurium growth in batch culture systems. FEMS Microbiol Ecol 66, 608-619.

45. Mountzouris KC, Balaskas C, Fava F, et al. (2006) Profiling of composition and metabolic activities of the colonic microflora of growing pigs fed diets supplemented with prebiotic oligosaccharides. Anaerobe 12, 178-185.
46. Loh G, Eberhard M, Brunner RM, et al. (2006) Inulin alters the intestinal microbiota and short-chain fatty acid concentrations in growing pigs regardless of their basal diet. J Nutr 136, 1198-1202.

47. Grieshop CM, Reese DE \& Fahey GC Jr (2000) Nonstarch polysaccharides and oligosaccharides in swine nutrition. In Swine Nutrition, pp. 107-130 [AJ Lewis and LL Southern, editors]. Boca Raton, FL: CRC Press.

48. Haenen D, Zhang J, Souza da Silva C, et al. (2013) A diet high in resistant starch modulates microbiota composition, SCFA concentrations, and gene expression in pig intestine. I Nutr 143, 274-283

49. Cleusix V, Lacroix C, Dasen G, et al. (2010) Comparative study of a new quantitative real-time PCR targeting the xylulose-5phosphate/fructose-6-phosphate phosphoketolase bifidobacterial gene $(x p p)$ in faecal samples with two fluorescence in situ hybridization methods. J Appl Microbiol 108, 181-193.

50. Furet JP, Firmesse O, Gourmelon M, et al. (2009) Comparative assessment of human and farm animal faecal microbiota using real-time quantitative PCR. FEMS Microbiol Ecol 68, 351-362.

51. Ramirez-Farias C, Slezak K, Fuller Z, et al. (2009) Effect of inulin on the human gut microbiota: stimulation of Bifidobacterium adolescentis and Faecalibacterium prausnitzii. Br J Nutr 101, 541-550.

52. Bartosch S, Fite A, Macfarlane GT, et al. (2004) Characterization of bacterial communities in feces from healthy elderly volunteers and hospitalized elderly patients by using real-time PCR and effects of antibiotic treatment on the fecal microbiota. Appl Environ Microbiol 70, 3575-3581.

53. Collado MC, Delgado S, Maldonado A, et al. (2009) Assessment of the bacterial diversity of breast milk of healthy women by quantitative real-time PCR. Lett Appl Microbiol 48, 523-528. 